\title{
Review
}

\section{Randomized Clinical Trials of Constitutional Acupuncture: A Systematic Review}

\author{
Myeong Soo Lee', Byung-Cheul Shin ${ }^{2}$, Sun-Mi Choi ${ }^{1}$ and Jong Yeol Kim ${ }^{1}$ \\ ${ }^{1}$ Korea Institute of Oriental Medicine, Daejeon and ${ }^{2}$ Department of Oriental Rehabilitation Medicine, School of \\ Oriental Medicine, Pusan National University, Busan, Korea
}

\begin{abstract}
The aim of this systematic review is to compile and critically evaluate the evidence from randomized clinical trials (RCTs) for the effectiveness of acupuncture using constitutional medicine compared to standard acupuncture. Ten databases were searched through to December 2008 without language restrictions. We also hand-searched nine Korean journals of oriental medicine. We included prospective RCTs of any form of acupuncture with or without electrical stimulation. The included trials had to investigate constitutional medicine. There were no restrictions on population characteristics. Forty-one relevant studies were identified, and three RCTs were included. The methodological quality of the trials was variable. One RCT found Sasang constitutional acupuncture to be superior to standard acupuncture in terms of the Unified PD Rating Scale and freezing gate in Parkinson's disease (PD). Another two RCTs reported favorable effects of eight constitutional acupuncture on pain reduction in patients with herniated nucleus pulposi and knee osteoarthritis. Meta-analysis demonstrated positive results for eight constitutional acupuncture compared to standard acupuncture on pain reduction (weighted mean difference: $10 \mathrm{~cm}$ VAS, $1.69,95 \%$ CI $0.85-2.54, P<0.0001$; heterogeneity: $\left.\tau^{2}=0.00, \chi^{2}=0.00, P=0.96, \mathrm{I}^{2}=0 \%\right)$. Our results provide suggestive evidence for the effectiveness of constitutional acupuncture in treating pain conditions compared to standard acupuncture. However, the total number of RCTs and the total sample size included in our analysis were too small to draw definite conclusions. Future RCTs should assess larger patient samples with longer treatment periods and appropriate controls.
\end{abstract}

Keywords: acupuncture - clinical trial - constitutional medicine - systematic review

\section{Introduction}

Korea has developed its own unique traditional medicine over time and established a different type of acupuncture from that used in traditional Chinese medicine. Korean acupuncture was developed using an individualized and practical approach, which is based on constitutional energy traits $(1,2)$. These traits have always been a main theme in Korean traditional medicine.

For reprints and all correspondence: Myeong Soo Lee, Korea Institute of Oriental Medicine, Daejeon, 305-811, South Korea. Tel: +82-42-8689266; Fax: +82-42-863-9464; E-mail: drmslee@gmail.com;

mslee@kiom.re.kr
Korean constitutional medicine has been represented with 'Sasang constitutional medicine'. This medicine was developed by Dr Je-ma Lee and published in the classic textbook Donguisusebowon (3). Sasang constitutional medicine classifies the human body into four types: Taeyangin (strong lung and weak liver), Soyangin (strong spleen and weak kidney), Taeeumin (strong liver and weak lung) and Soeumin (strong kidney and weak spleen). Sasang constitutional medicine distinguishes an individual's type according to differences in four major organs (liver, lung, spleen and kidneys) (3). Different interventions are applied according to a person's specific type including both the component of 'the physical body' and the aspect of 'the functional system' (4). 
The morbidity of a given constitution in Sasang constitutional medicine may be caused by the excessively strong function of the strongest organ or by the excessively weak function of the weakest organ (2).

Eight constitutional medicine is a subdivision of Sasang constitutional medicine. The theory behind eight constitution acupuncture is that each of the four constitutions described in Sasang constitutional medicine are further subdivided into two constitutions; Metal Yin/ Yang, Earth Yin/Yang, Wood Yin/Yang and Water Yin/Yang (2).

There are several types of acupuncture that use constitutional medicine, including Taegeuk acupuncture and eight constitutional acupuncture (2). Taegeuk acupuncture applies Sasang constitutional medicine and considers the four constitutions as underlying entities (2). Eight constitutional medicine suggests that there are eight constitutional traits that are the core determinants of physiologic or pathologic processes, and it provides remedies including acupuncture points (eight constitutional acupuncture) based on these constitutional traits (2). Comparing to constitutional acupuncture, standard (or classic) acupuncture is based on meridian theory following the individualized person's symptoms of disease, called pattern identification (or syndrome differentiation).

Recently published reviews summarized the clinical effectiveness of Korean acupuncture $(1,2,5)$. They summarized the characteristics of acupuncture in Korean traditional medicine as individualized and practical acupuncture. However, these reviews did not focus on the effects of constitutional acupuncture. Furthermore, none of the reviews attempted to compare constitutional acupuncture and standard acupuncture. The aim of this systematic review is to synthesize and critically appraise the evidence from RCTs for the effectiveness of acupuncture using constitutional medicine compared to standard acupuncture.

\section{Methods}

\section{Database and Search Strategies}

We searched electronic databases from inception through to December 2008 using the following databases: MEDLINE, CINAHL, EMBASE, PsycInfo, The Cochrane Library 2008 (Issue 4) and five Korean medical databases (Korean Studies Information, DBPIA, Korea Institute of Science and Technology Information, KERIS and KoreaMed). We used search terms based on two concepts. The first concept included all terms for 'acupuncture', and the second concept included all terms for 'constitution'. The two concepts were combined using the Boolean operator 'AND'. Korean terms for 'constitution' and 'acupuncture' were used in the Korean databases. In addition, we hand-searched nine Korean traditional oriental medical journals [Journal of Sasang Constitutional Medicine, Journal of Oriental Medicine, The Journal of Korean Acupuncture \& Moxibustion Society, The Korean Journal of Meridian \& Acupoint, Journal of Oriental Rehabilitation Medicine, Journal of Korean Institute of Herbal Acupuncture, The Journal of Korea Chuna Manual Medicine, Korean Journal of Oriental Physiology \& Pathology and The Journal of Korean Oriental Internal Medicine (searched from their inception through December 2008)].

\section{Study Selection}

We included prospective RCTs of any form of acupuncture including electro-acupuncture. All studies had to include constitutional medicine. There were no restrictions on population characteristics. The main outcome measure was the efficacy of constitutional medicine acupuncture when compared to standard acupuncture. No language restrictions were imposed. Dissertations and abstracts were included. Hard copies of all articles were obtained and read in full.

\section{Data Extraction and Quality Assessment}

Data were extracted systematically in a predefined, standardized manner according to study design, number of subjects, intervention and control groups (Table 1). The Jadad score was calculated using three criteria, which included description of randomization, blinding and withdrawals. The Jadad score ranges from 0 to 5 points $(6,7)$. Taking into account that patients and therapists are virtually impossible to be blinded to acupuncture, one point was given if the outcome assessor was blinded. Allocation concealment was assessed using the Cochrane Collaboration Classification (8). Selection, data extraction and quality assessment were performed independently by two reviewers (MSL, BCS). Discrepancies were resolved by discussion between the two reviewers.

\section{Data Synthesis}

The mean change in each of the outcome measures was compared. A baseline was used to assess the differences between the intervention groups and the control groups. Weighted mean differences (WMD) and 95\% confidence intervals (CI) were calculated using Cochrane Collaboration's software [Review Manager (RevMan) Version 5.0 for Windows. Copenhagen: The Nordic Cochrane Centre]. We then pooled the data across studies using a random effects model. The chi-squared test and the Higgins $\mathrm{I}^{2}$ test were used to assess heterogeneity. 


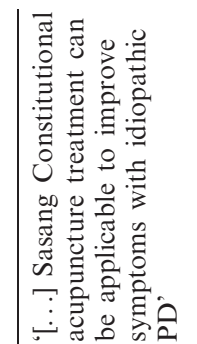

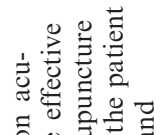

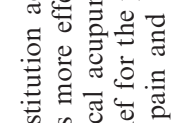

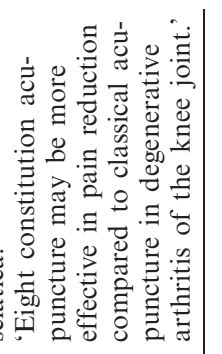

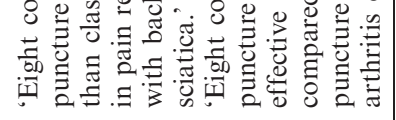
๓

की

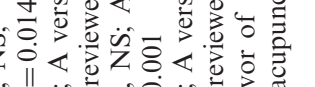

نरिज记

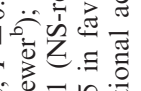

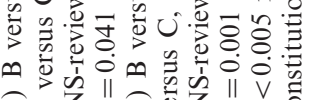

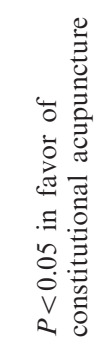

产

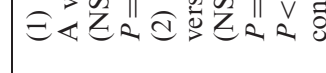

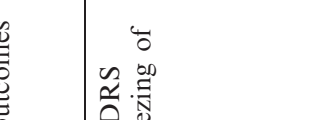

产高䓵

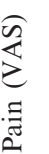

$\sum_{\substack{\pi \\ 0}}^{0}$

空官 $N$

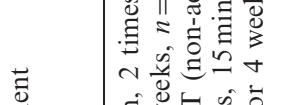

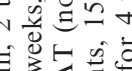

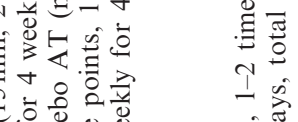

宫
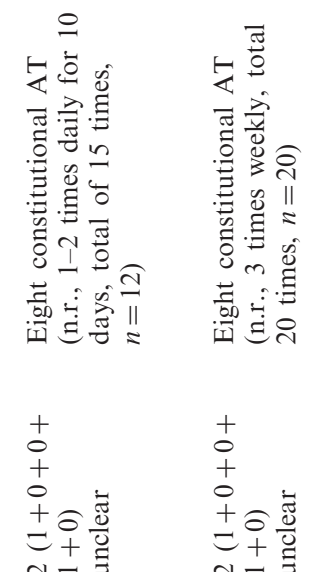

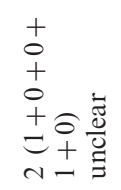

\section{N-}

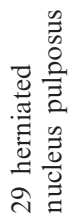

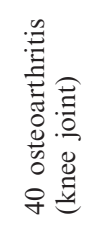

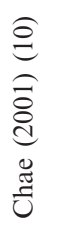

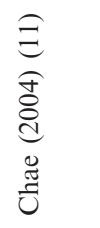

毒

爰.

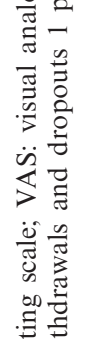




\section{Results}

\section{Study Description}

Our searches identified 41 potentially relevant articles, 38 of which were excluded. The excluded articles included 1 duplicate study and 37 trials unrelated to constitutional acupuncture. Three RCTs met our inclusion criteria (9-11). The key data are summarized in Table 1. One RCT (9) employed Sasang constitutional acupuncture, and two RCTs $(10,11)$ used Eight constitutional acupuncture. The treated conditions were Parkinson's disease (PD, one trial) (9), herniated nucleus pulposus (HNP, one trial) (10) and osteoarthritis of the knee joint (OA, one trial) (11). The employed acupuncture points are summarized in Table 2.

\section{Study Quality}

The methodology of the trials was variable. One of the RCTs described the methods of randomization (9) and all of the included RCTs employed subjects' blinding (9-11). Sufficient details of dropouts and withdrawals were described in one report (9). None of the reports described allocation concealment, assessor blind and adverse events. Ethical approval procedures were mentioned in one RCT (9).

\section{Outcomes}

Park et al. (9) conducted an RCT to assess the effectiveness of Sasang constitutional acupuncture on symptoms of PD. Fifty-five PD patients were divided randomly into three parallel groups: Sasang constitutional acupuncture

Table 2. Summary of treated acupuncture points

\begin{tabular}{|c|c|c|}
\hline First author (year) & Experimental group & Control group \\
\hline Park (2007) (9) & $\begin{array}{l}\text { (1) Soyangin: KI17(T), LR2(S), TE6(S) } \\
\text { (2) Taeyangin: LR8(T), HT7(S), SP3(S) } \\
\text { (3) Soeumin: SP2(T), LU5(S), KI10(S) } \\
\text { (4) Taeeumin: LU9(T), KI1(S), LR1(S) }\end{array}$ & LR3, GB34, ST36 \\
\hline Chae (2001) (10) & $\begin{array}{l}\text { Basic prescription (5 times) } \\
\text { (1) Metal-Yang: LU8(S), LR4 (S), KI10(T), LR8(T) } \\
\text { (2) Metal-Yin: KI10(S), LU5(S), LR1(T), LU11(T) } \\
\text { (3) Earth-Yang: SP3(S), KI3(S), LU8(T), KI7(T) } \\
\text { (4) Earth-Yin: LU8(S), SP5(S), KI10(T), SP9(T) } \\
\text { (5) Wood-Yang: LR8(S), KI10(S), LU8(T), LR4(T) } \\
\text { (6) Wood-Yin: KI10(T), LU5(T), LR1(S), LU11(S) } \\
\text { (7) Water-Yang: SP3(T), KI3(T), LU8(S), KI7(S) } \\
\text { (8) Water-Yin: LU8(T), SP5(T), KI10(S), SP9(S) } \\
\text { Supplemented prescription-Organ System Inflammation (5 times) } \\
\text { (1) Metal-Yang: SP3(S), LU9(S), LR1(T), LU11(T) } \\
\text { (2) Metal-Yin: LR4(S), LU8(S), HT8(T), LR2(T) } \\
\text { (3) Earth-Yang: HT8(S), SP2(S), KI10(T), SP9(T) } \\
\text { (4) Earth-Yin: SP3(S), KI3(S), LR1(T), KI1(T) } \\
\text { (5) Wood-Yang: SP3(T), LU9(T), LR1(S), LU11(S) } \\
\text { (6) Wood-Yin: LR4(T), LU8(T), HT8(S), LR2(S) } \\
\text { (7) Water-Yang: HT8(T), SP2(T), KI10(S), SP9(S) } \\
\text { (8) Water-Yin: SP3(T), KI3(T), LR1(S), KI1(S) } \\
\text { Supplemented prescription-Spirit (1 time) } \\
\text { (1) Metal-Yang: PC7(S), PC3(T) } \\
\text { (2) Metal-Yin: SI1(S), SI3(T) } \\
\text { (3) Earth-Yang: HT7(S), HT3(T) } \\
\text { (4) Earth-Yin: TE1(S), TE3(T) } \\
\text { (5) Wood-Yang: PC7(T), PC3(S) } \\
\text { (6) Wood-Yin: SI1(T), SI3(S) } \\
\text { (7) Water-Yang: HT7(T), HT3(S) } \\
\text { (8) Water-Yin: TE1(T), TE3(S) }\end{array}$ & $\begin{array}{l}\text { Bilateral: BL23, BL24, BL25, BL26 } \\
\text { Some of bilateral: BL31-34, } \\
\text { Pain side of GB30, BL40, BL57, BL60 }\end{array}$ \\
\hline Chae (2004) (11) & $\begin{array}{l}\text { Basic prescription (5 times) } \\
\text { Same as Chae (10) } \\
\text { Supplemented prescription-Vitality (5 times) } \\
\text { (1) Metal-Yang: ST36(S), LI11(S), GB41(T), LI3(T) } \\
\text { (2) Metal-Yin: LI1(S), GB44(S), SI5(T), GB38(T) } \\
\text { (3) Earth-Yang: SI5(S), ST41(S), BL66(T), ST44(T) } \\
\text { (4) Earth-Yin: ST36(S), BL40(S), GB41(T), BL65(T) } \\
\text { (5) Wood-Yang: ST36(T), LI11(T), GB41(S), LI3(S) } \\
\text { (6) Wood-Yin: LI1(T), GB44(T), SI5(S), GB38(S) } \\
\text { (7) Water-Yang: SI5(T), ST41(T), BL66(S), ST44(S) } \\
\text { (8) Water-Yin: ST36(T), BL40(T), GB41(S), BL65(S) } \\
\text { Supplemented prescription-Spirit (1 times) } \\
\text { Same as Chae (10) }\end{array}$ & $\begin{array}{l}\text { Heding (Extra), Xiyan (internal \& external, Extra), } \\
\text { SP9, GB34, LR3, SP10, ST34, ST36 }\end{array}$ \\
\hline
\end{tabular}

S: Sedation, T: Tonification. 


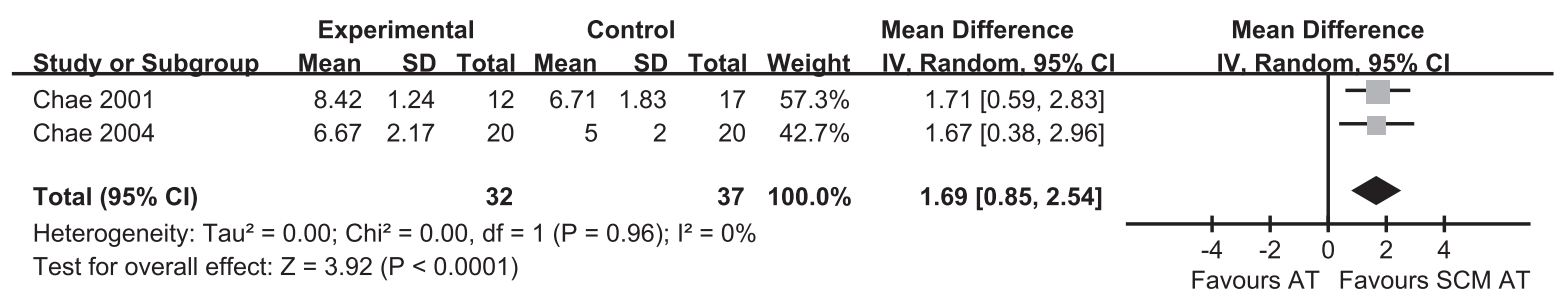

Figure 1. Forest plot of constitutional acupuncture for pain conditions (eight constitutional acupuncture, EC AT versus standard acupuncture, AT).

$(n=12)$, standard acupuncture $(n=21)$ and sham control (needle penetration at non-acupuncture sites) $(n=13)$. The outcome measures included the Unified PD Rating Scale (UPDRS) and freezing gait. At the end of the treatment period, Sasang constitutional acupuncture showed favorable effects on UPDRS and freezing gait compared to standard acupuncture and sham control.

Chae et al. (10) tested the effect of eight constitutional acupuncture on pain in HNP patients. Twentynine patients were randomly assigned to two treatment groups: eight constitutional acupuncture $(n=12)$ or standard acupuncture $(n=17)$. After 10 days of treatment, the eight constitutional acupuncture group reported significantly greater pain reduction than the standard acupuncture group.

Chae and Song (11) investigated the effects of eight constitutional acupuncture on pain reduction in patients with OA. Patients were randomized into two groups: one receiving eight constitutional acupuncture $(n=20)$ and the other receiving standard acupuncture $(n=20)$. After treatment, the pain reduction was significantly better in the eight constitutional acupuncture group than in the control group.

\section{Pain Reduction}

Two RCTs $(10,11)$ compared the effects of eight constitutional acupuncture with standard acupuncture in pain conditions. Meta-analysis showed favorable effects of eight constitutional acupuncture on pain improvement $(n=69$, WMD, $10 \mathrm{~cm}$ VAS, $1.69,95 \%$ CI $0.85-2.54$, $P<0.0001$; heterogeneity: $\tau^{2}=0.00, \chi^{2}=0.00, P=0.96$, $\mathrm{I}^{2}=0 \%$, Fig. 1).

\section{Discussion}

The most important finding of this systematic review is that there are very few rigorous trials comparing the effects of constitutional acupuncture and standard acupuncture. Our systematic review and meta-analysis provides evidence that constitutional acupuncture is more effective than standard acupuncture in the treatment of pain conditions $(10,11)$. However, the total number of RCTs and the total sample size included in our analysis were too small to draw firm conclusions about the superiority of constitutional acupuncture.

We assessed study quality using a modified Jadad scale. One point was awarded for subject blinding or assessor blinding, respectively. Two of the three trials had a methodology quality score of ' 2 ' on this scale $(10,11)$. Low-quality trials are more likely to overestimate the effect size (12). All three trials employed patient blinding, though none attempted to blind the assessors. None of the trials used allocation concealment. Trials with inadequate blinding and inadequate allocation concealment are likely to have exaggerated effects (12). All of the trials had small sample sizes, which reduced the possibility of drawing a meaningful conclusion. No power analysis was included.

One trial of Sasang constitutional acupuncture suggested positive effects on symptoms of PD (9). However, this study did not compare the baseline values of outcomes and those seemed to have large differences among the groups. The baseline imbalances may lead to erroneous conclusions from the statistical analyses. Moreover, this study used an inappropriate statistical analysis. The use of ANCOVA instead of $t$-test or ANOVA might overestimate the effects of acupuncture. The effectiveness of Sasang constitutional acupuncture for PD has thus not been convincingly demonstrated.

One area of concern is the design of the included trials. The comparison of standard acupuncture to constitutional medicine-based acupuncture style may not be appropriate for some conditions or may be meaningless. Future studies determining the efficacy of constitutional acupuncture should include appropriate sham acupuncture in addition to standard acupuncture. The third concern is that ethical approval was reported in only one trial (9). Researchers must conduct their research in an ethical manner. Moreover, clinical trials should follow CONSORT procedures (13).

This systematic review has several limitations. The first is that relevant RCTs might have been overlooked despite our best efforts to conduct sensitive literature searches without language restrictions. Second, all three of the included RCTs were conducted in a single country. Studies from South Korea are known to exhibit a very low rate of negative results (14). The distorting effects of publication bias and location bias are well 
documented (15-17). Therefore, independent replications are required, preferably in other countries. Another weakness of our systematic review pertains to the quality of the primary researches. The reviewed studies were of moderate quality; however, methodological shortcomings such as small sample sizes and inadequate blinding require further larger studies incorporating adequate blinding procedures.

Future trials testing the effectiveness of constitutional acupuncture should adhere to rigorous trial designs that are suitable for research. Such trials should preferably be randomized and double blinded (including assessor blinding), control for placebo effects, have adequate allocation concealment, have sample sizes based on appropriate sample-size calculations, use validated outcome measures, and include a full description of the actual interventions being tested. They should also follow CONSORT procedures. Furthermore, one of the most important prerequisites of any constitutional medicine is accuracy of diagnosis of each independent constitution. The issue of the reliability in constitutional medicine needs further attention in future constitutional medicine research.

In conclusion, the results of our systematic review and meta-analysis suggest that constitutional acupuncture is superior to standard acupuncture in the treatment of pain conditions. However, the total number of RCTs included in the analysis and the total sample size were too small to draw firm conclusions about the effectiveness of this approach. Future RCTs should assess larger patient samples for longer treatment periods and include appropriate controls.

\section{Perspective}

Constitutional acupuncture involves selecting acupuncture points according to persons' specific physical and physiological type and is used in many conditions. A metaanalysis produced limited evidence for the effectiveness of constitutional acupuncture in musculoskeletal pain management. However, primary data were scarce. Future RCTs should assess larger patient samples for longer treatment periods and include appropriate controls.

\section{Acknowledgement}

The authors specially thank Kate Boddy of Peninsula Medical School, Universities of Exeter \& Plymouth, Exeter, UK for comments and editing this manuscript.

\section{Funding}

Korea Institute of Oriental Medicine (K09050); Korea Science and Engineering Foundation (KOSEF) and its grant funded by the Korea Ministry of Education, Science and Technology (MEST) (No. M1064302000108N4302-00100).

\section{References}

1. Kim YS, Jun H, Chae Y, Park HJ, Kim BH, Chang IM, et al. The practice of Korean medicine: an overview of clinical trials in acupuncture. eCAM 2005;2:325-52.

2. Yin C, Park HJ, Chae Y, Ha E, Park HK, Lee HS, et al. Korean acupuncture: the individualized and practical acupuncture. Neurol Res 2007;29(Suppl 1):S10-5.

3. Lee J. Donguisusebowon. Korea: Seoul, 1894.

4. Lee J, Jung Y, Yoo J, Lee E, Koh B. Pespective of the human body in Sasang Constitutional Medicine. eCAM 2009;6(Suppl 1).

5. Kong JC, Lee MS, Shin B-C. Randomized clinical trials on acupuncture in Korean literature: a systematic review. eCAM 2009;6:41-8.

6. Jadad AR, Moore RA, Carroll D, Jenkinson C, Reynolds DJ, Gavaghan DJ, et al. Assessing the quality of reports of randomized clinical trials: is blinding necessary? Control Clin Trials 1996; $17: 1-12$.

7. White AR, Ernst E. A systematic review of randomized controlled trials of acupuncture for neck pain. Rheumatology 1999;38:143-7.

8. Higgins JPT, Greens S (eds). Cochrane Handbook for Systematic Reviews of Interventions 4.2.6 [updated September 2006]. Available from Cochrane Library, Issue 4, 2006. Chichester, UK: John Wiley \& Sons, Ltd., 2006.

9. Park YC, Chang DI, Lee YH, Park DS. The study on the effect of acupuncture treatment in patients with idiopathic Parkinson's disease. $J$ Korean Acupunct Moxibust Soc 2007;24:43-54 (in Korean).

10. Chae SJ, Kim NO, Park YC, Son SS. Comparison of the improvement of subjective symptoms between body acupuncture group and 8 constitution acupuncture group. J Korean Acupunct Moxibust Soc 2001;18:48-55 (in Korean).

11. Chae SJ, Song HS. The effect of 8 constitution acupuncture on degenerative arthritis of knee joint. $J$ Korean Acupunct Moxibust Soc 2004;21:65-73 (in Korean).

12. Schulz KF, Chalmers I, Hayes RJ, Altman DG. Empirical evidence of bias. Dimensions of methodological quality associated with estimates of treatment effects in controlled trials. JAMA 1995;273: $408-12$.

13. Begg C, Cho M, Eastwood S, Horton R, Moher D, Olkin I, et al. Improving the quality of reporting of randomized controlled trials. The CONSORT statement. JAMA 1996;276:637-9.

14. Vickers A, Goyal N, Harland R, Rees R. Do certain countries produce only positive results? A systematic review of controlled trials. Control Clin Trials 1998;19:159-66.

15. Dickersin K. The existence of publication bias and risk factors for its occurrence. JAMA 1990;263:1385-9.

16. Egger M, Smith GD. Bias in location and selection of studies. Br Med J 1998;316:61-6.

17. Ernst E, Pittler MH. Alternative therapy bias. Nature 1997;385:480. 


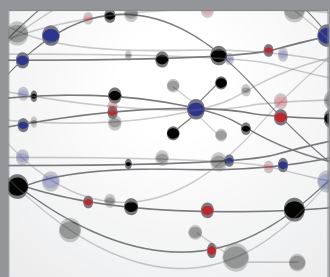

The Scientific World Journal
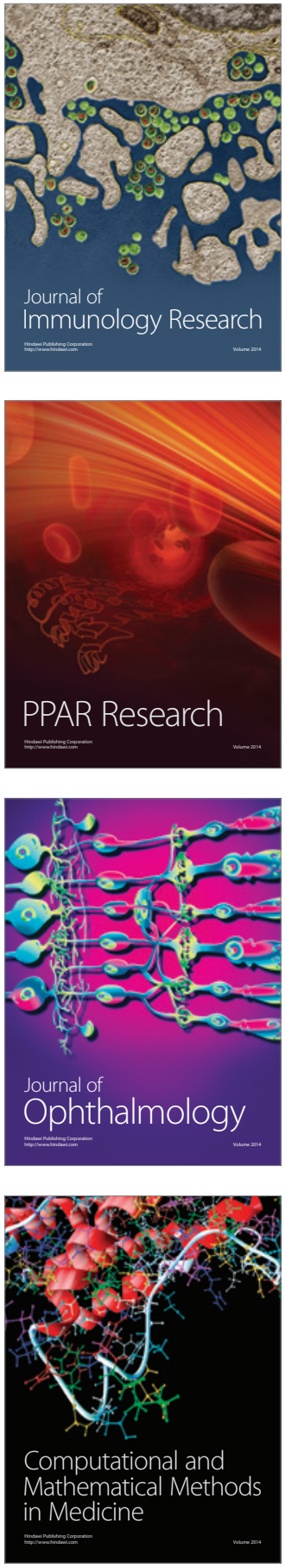

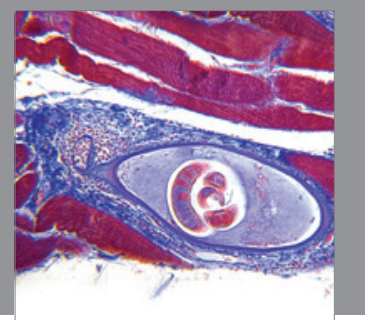

Gastroenterology

Research and Practice
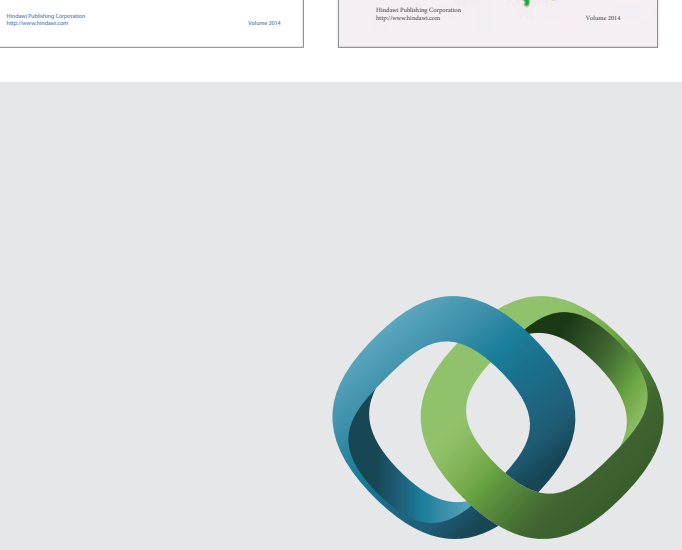

\section{Hindawi}

Submit your manuscripts at

http://www.hindawi.com
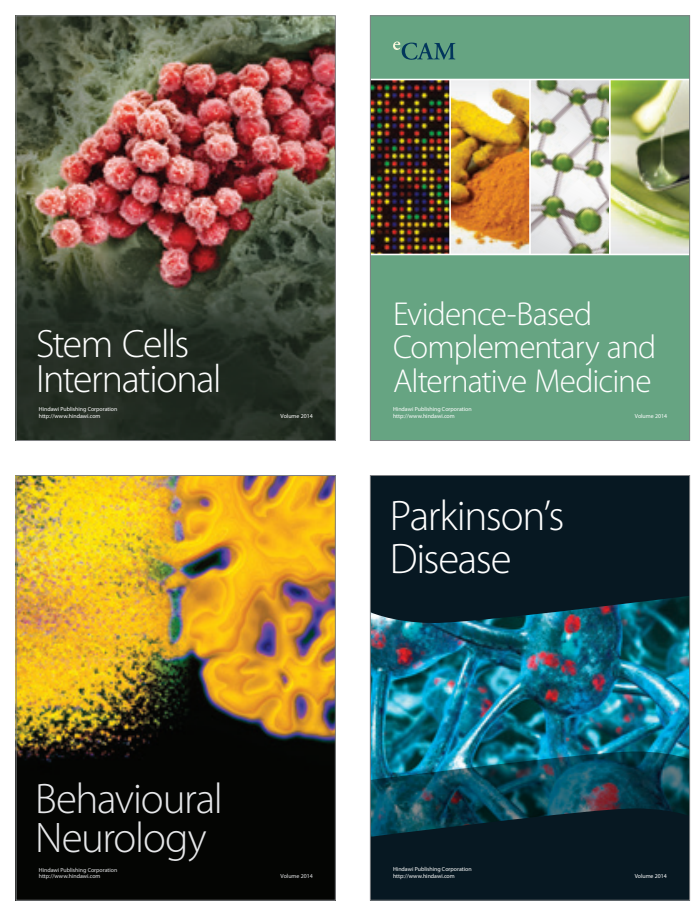

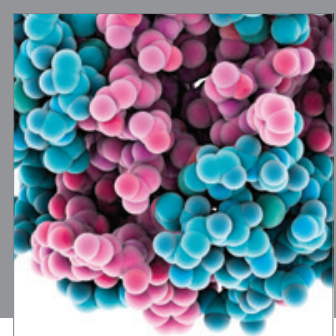

Journal of
Diabetes Research

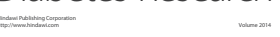

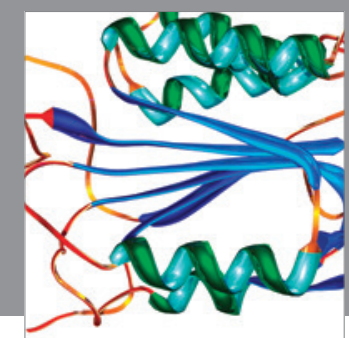

Disease Markers
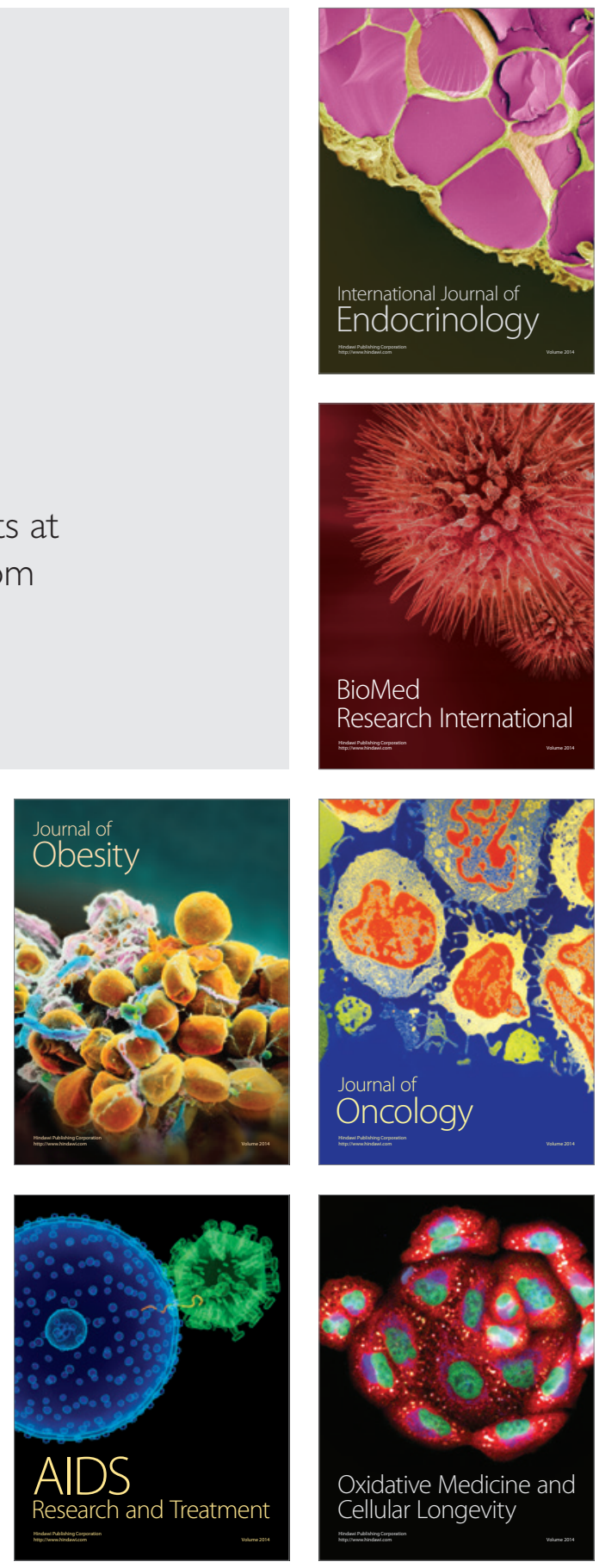